\title{
Frequency, Aetiology, and Outcome of Small Cerebellar Infarction
}

\author{
Zeljka Calic $^{\mathrm{a}-\mathrm{d}}$ Cecilia Cappelen-Smith ${ }^{\mathrm{a}-\mathrm{c}}$ Ramesh Cuganesan ${ }^{\mathrm{e}}$ \\ Craig S. Anderson ${ }^{d, f}$ Miriam Welgampola $^{f}$ Dennis J. Cordato ${ }^{a-c}$ \\ a Department of Neurology and Neurophysiology, Liverpool Hospital, Liverpool, NSW, \\ Australia; ${ }^{b}$ Ingham Institute for Applied Medical Research, Liverpool, NSW, Australia; \\ 'South Western Sydney Clinical School, University of New South Wales, Sydney, NSW, \\ Australia; ${ }^{d}$ The George Institute for Global Health, Faculty of Medicine, University of \\ New South Wales, Sydney, NSW, Australia; ' Department of Radiology, Liverpool Hospital, \\ Liverpool, NSW, Australia; ${ }^{f}$ Institute of Clinical Neurosciences, Royal Prince Alfred Hospital, \\ Central Clinical School, University of Sydney, Sydney, NSW, Australia
}

Keywords
Cerebellar infarction · Aetiology $\cdot$ Topography $\cdot$ Outcome

\section{Abstract}

Background and Purpose: Strokes due to small $(<2 \mathrm{~cm})$ cerebellar infarction are under-recognised, and their profile and aetiology have not been well characterised. We aimed to determine the frequency, clinical features, aetiology, and outcome of small as compared to large cerebellar infarction. Methods: This study is a retrospective analysis of clinical and imaging features of a prospectively assessed series of 108 consecutive patients with acute cerebellar infarction admitted to Liverpool Hospital, Sydney, NSW, Australia, during 2011-2015. Results: The mean age of the patients was 67 years, and 33 (31\%) had small cerebellar infarction. Compared to large cerebellar infarction, those with small cerebellar infarction had a comparable distribution of vascular risk factors but significantly less nausea and vomiting, gait disturbance, limb ataxia, and dysarthria. The posterior $(n=22,67 \%)$ lobe was most commonly affected, followed by the anterior $(n=9,27 \%)$ and flocculonodular $(n=2)$ lobes. Dizziness, limb ataxia, and nystagmus were significantly more common in patients with anterior lobe infarction. Vertebrobasilar disease was the presumed aetiology in 40 patients (37\%), and was less commonly seen in small as compared to large cerebellar infarction. Cardioembolism affected $37 \%$ of the patients, irrespective of the size or topography of the cerebellar infarction, and there was no relation of supratentorial white matter lucencies (WMLs) to the size of cerebellar infarction. At 3 months, 65\% of the patients were functionally independent (according to modified Rankin Scale scores of $0-2$ ), and having a poor outcome was significantly related to 
moderate-to-severe supratentorial WML and large cerebellar infarction. Conclusions: Small cerebellar infarction accounted for one-third of the ischaemic strokes in this location, most often involved the posterior lobe, causing fewer clinical features, and had a better clinical outcome than large cerebellar infarction. Patients with small cerebellar infarction require appropriate vascular management including investigation for a cardioembolic source.

(C) 2017 The Author(s)

Published by S. Karger AG, Basel

\section{Introduction}

Cerebellar infarction (CBI) accounts for $2-3 \%$ of all ischaemic strokes [1, 2], and can be classified as small or large on the basis of a 2 -cm cut point in diameter [3, 4]. The primary functions of the cerebellum are sensorimotor (anterior lobe), non-motor affective and cognitive (posterior lobe), vestibular (flocculonodular lobe), and mixed sensorimotor, cognitive, and limbic processing within the deep white matter (Fig. 1) [5, 6]. Uncertainty exists over how the aetiology and outcome are related to the topography of small CBI, in particular with regard to cerebral small vessel disease, which most clearly manifests as supratentorial white matter lucencies (WMLs) [7-9]. One study of isolated first-ever CBI suggests an association of severity of supratentorial WML with cerebellar symptoms [10].

We aimed to determine the frequency, aetiology, and outcome of acute small CBI as compared to large CBI; the relation of clinical features and aetiology to the lesion topography of small CBI; and any association of supratentorial WML with aetiology and outcome of small CBI.

\section{Methods}

This study is a subanalysis of a previously published prospective cohort study of a consecutive series of 115 patients with acute CBI who were admitted to Liverpool Hospital, a large teaching and tertiary referral hospital in Sydney, NSW, Australia, from November 2011 to August 2015 [1]. Infarct size was classified on axial MRI diffusion-weighted imaging sequences $(n=92)$ or axial CT scans $(n=16)$ as small $(\leq 2 \mathrm{~cm})$ or large $(>2 \mathrm{~cm})$ on the basis of the longest diameter of a single area. Patients who only underwent CT had a contraindication to MRI, which included having a low Glasgow Coma Scale score $(n=10)$, delirium $(n=5)$, or a pacemaker $(n=1)$. All patients with multiple CBIs had typically bilateral CBIs $(n=7)$. Due to uncertainty of classification, patients with multiple CBIs were excluded from our study. Ethics approval was obtained from the South Western Sydney Local Health District Human Research Ethics Committee.

Demographic characteristics, medical histories, and presenting clinical features were obtained. All patients were assessed by a neurology-trained physician within $4 \mathrm{~h}$ of their assessment in the ED (or immediately if a stroke call was activated) if their presentation was during weekday working hours (8 a.m. to 5 p.m.) $(n=52)$, and within $24 \mathrm{~h}$ of an ED assessment if their presentation was "after" hours, unless a stroke call was activated ( $n=56$, including 18 weekend presentations). The topography of small CBI, extent of supratentorial WML, and presence of vertebrobasilar disease (VBD) were independently assessed by at least 2 of the co-authors, with results determined by consensus if disagreement existed. Vascular territories were determined in accordance with the diagrams by Amarenco et al. [3]. A small CBI topography was determined from T2 axial MRI sequences $(n=32)$ or a CT scan $(n=1)$ and categorised using the functional topographic classification proposed by De Cocker et al. [5] 


\section{Cerebrovascular" \\ Diseases}

Fig. 1. Axial $\mathrm{T} 2$-weighted $\mathrm{MR}$ images. a Medullar/cerebellar cross-section showing fissures (dotted lines) between lobules VII, VIII, and IX. b Mid-pontine/ cerebellar cross-section showing a primary fissure (PF, black lines) dividing the cerebellum into anterior and posterior lobes, and separating lobules V and VI. The posterior superior fissure (PSF, white lines) separates lobules VI and VII, and the great horizontal fissure (GHF, white lines) separates lobule VII (crus [Cr] I) from lobule VII (Cr II). c Rostral pontine/ cerebellar cross-section showing lobules I-VII and fissures separating these lobules.

\begin{tabular}{l|l}
\hline Cerebrovasc Dis Extra 2017;7:173-180 \\
\hline DOI: 10.1159/000481459 & $\begin{array}{l}\text { @ } 2017 \text { The Author(s). Published by S. Karger AG, Basel } \\
\text { www.karger.com/cee }\end{array}$ \\
\hline
\end{tabular}

Calic et al.: Frequency, Aetiology, and Outcome of Small Cerebellar Infarction
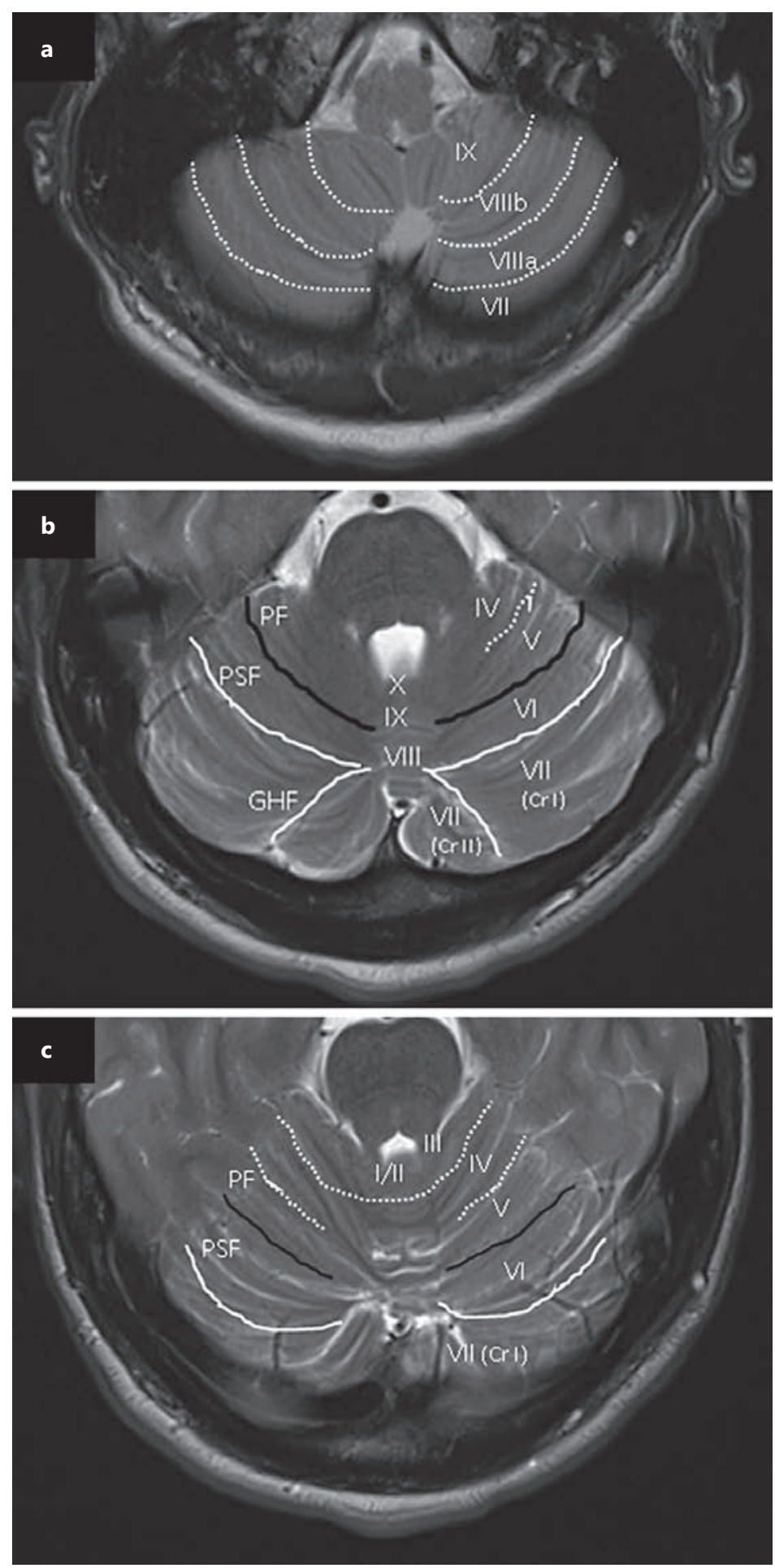

and the Schmahmann diagrams [11]. Small CBIs were classified into anterior lobe (lobules I-V), posterior lobe (lobules VI-IX), and flocculonodular lobe (lobule X) (Fig. 1). The presence of presumed symptomatic VBD (excluding dissection) was determined by MR and/or CT angiography $(n=90)$ and defined as stenosis $\geq 50 \%$ or complete vessel occlusion. The presence of a dominant vertebral artery, defined by a larger diameter on visual inspection, 
Table 1. Characteristics of the patients with small and large CBI

\begin{tabular}{lccc}
\hline & $\begin{array}{l}\text { Small CBI } \\
(n=33)\end{array}$ & $\begin{array}{l}\text { Large CBI } \\
(n=75)\end{array}$ & $p$ value \\
\hline $\begin{array}{l}\text { Demography } \\
\text { Age, years }\end{array}$ & $70 \pm 11$ & $66 \pm 14$ & 0.10 \\
Male & $14(42)$ & $41(55)$ & 0.24 \\
History & $24(73)$ & $53(71)$ & 0.82 \\
$\quad$ Hypertension & $24(73)$ & $47(63)$ & 0.45 \\
Hypercholesterolaemia & $15(45)$ & $21(28)$ & 0.07 \\
Diabetes mellitus & $9(27)$ & $23(31)$ & 0.72 \\
Atrial fibrillation & $9(27)$ & $19(25)$ & 0.72 \\
Current smoking & $9(27)$ & $16(21)$ & 0.50 \\
Prior stroke/TiA & $5(15)$ & $12(16)$ & 0.89 \\
Family history of stroke & $20(61)$ & $54(72)$ & 0.24 \\
Presenting symptoms & $17(52)$ & $55(73)$ & 0.03 \\
Dizziness & $13(39)$ & $54(72)$ & 0.001 \\
Gait disturbance & $12(36)$ & $35(47)$ & 0.32 \\
Nausea and vomiting & $9(27)$ & $35(47)$ & 0.05 \\
Vertigo & & & \\
Headache & $9(27)$ & $45(66)$ & 0.001 \\
Presenting signs & $10(31)$ & $33(48)$ & 0.10 \\
Limb ataxia & $3(9)$ & $25(37)$ & 0.01 \\
Nystagmus & $1(3)$ & $13(19)$ & 0.06 \\
Dysarthria & &
\end{tabular}

Data are the mean \pm SD or $n(\%)$. Italic type denotes significance. CBI, cerebellar infarction; TIA, transient ischaemic attack. ${ }^{1}$ Data pertaining to 100 patients, as 8 were excluded due to being intubated and sedated after presenting comatose and with respiratory compromise.

was also examined. The extent of supratentorial WML, determined in patients who underwent MRI with T2 flair sequences $(n=92)$, was dichotomised into "nil or mild" (score $0-1)$ or "moderate to severe" (score 2-3) [12].

A presumed cardioembolic cause of CBI was based on the presence of any prior or newonset atrial fibrillation, left ventricular thrombus, or severe dysfunction (defined as an ejection fraction of $<30 \%$ on transthoracic echocardiography). All patients had their outcome assessed using the modified Rankin Scale (mRS) at 3 months, with functional independence defined as a score of $0-2$.

Associations of continuous variables were evaluated using the independent-sample $t$ test, and categorical variables using the $\chi^{2}$ test. Independent associations were assessed with binary logistic regression including variables that were significant $(p<0.05)$ in univariate analysis, with data reported with odds ratios (ORs) and 95\% confidence intervals (CIs). Statistical analyses were performed with SPSS software version 23 for Windows (SPSS, Inc., Chicago, IL, USA).

\section{Results}

Of the 108 patients (age, mean \pm SD $67 \pm 13$ years, range 32-92; 51\% male), $51(47 \%)$ had isolated CBI and 33 (31\%) had small CBI, which was isolated to the cerebellum in 17 cases. Twenty-four patients (22\%) underwent their initial CT within $4.5 \mathrm{~h}$ and $50(46 \%)$ 


\section{Cerebrovasculai \\ Diseases}

Fig. 2. Axial diffusion-weighted MR images showing acute cerebellar infarction (CBI). a Large CBI involving the left cerebellum. b Small CBI involving the right anterior lobe. c Small CBI involving the flocculonodular lobe. d Small CBI involving the left posterior lobe.

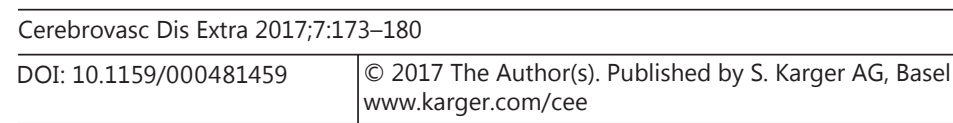

Calic et al.: Frequency, Aetiology, and Outcome of Small Cerebellar Infarction
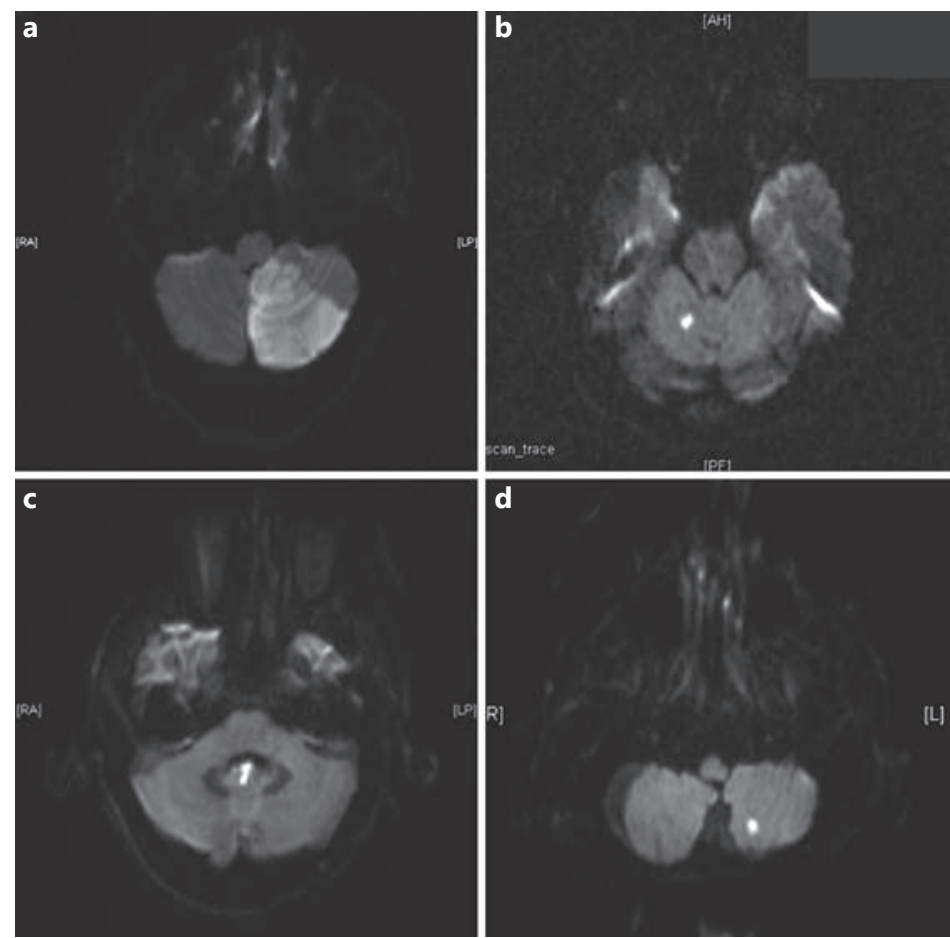

between 4.5 and $24 \mathrm{~h}$ from symptom onset. Twelve patients (11\%) had an initial CT between 24 and $48 \mathrm{~h}$ and $22(21 \%)$ after $48 \mathrm{~h}$ from symptom onset. MRI scans were performed within $24 \mathrm{~h}$ in only 3 patients, within $24-48 \mathrm{~h}$ in 24 patients (26\%), and after $48 \mathrm{~h}$ from symptom onset in 65 patients $(71 \%)$. The 15 patients who underwent CT alone due to having a contraindication to MRI all had large CBIs, with 7 dying in hospital and 3 being functionally dependent at 3 months.

Table 1 shows that the patients with small and large CBIs had a comparable distribution of vascular risk factors and clinical features except for less nausea and vomiting, gait disturbance, limb ataxia, and dysarthria among those with small CBIs.

The cerebellar vascular territory most commonly involved was the posterior inferior cerebellar artery (PICA) in 64 (59\%) of the patients, followed by the superior cerebellar artery (SCA) in 20 patients (19\%) and the anterior inferior cerebellar artery in 2 patients (2\%). Two or more cerebellar vascular territories were involved in 22 patients $(20 \%)$. There was no difference in clinical features between CBI involving the PICA and CBI involving the SCA.

The topographical distribution of small CBIs was in the posterior ( $n=22,67 \%)$, anterior $(n=9,27 \%)$, and flocculonodular $(n=2)$ lobes (Fig. 2). Thirteen small CBIs involved the border zone or immediate vicinity of the primary fissure $(n=6$ in lobule $\mathrm{V}$ of the anterior lobe and $n=7$ in lobule VI of the posterior lobe) [5]. There were no cases of small CBI involving the deep cerebellar white matter or nuclei. Dizziness, limb ataxia, and nystagmus were significantly more frequent in patients with anterior lobe involvement. No other presenting symptoms and signs had a significant lobar predilection.

Significant VBD was the presumed aetiology in 33/57 (58\%) of the patients with large CBI and 7/33 (23\%) of the patients with small CBI. There was a diversity of atheromatous VBD in the small CBIs (4 with stenosis or occlusion of the basilar artery, 2 with occlusion of the vertebral artery, and 1 with occlusion of the PICA) and 2 patients with small CBI had dissection of the vertebral artery. Significant VBD was less common in small compared to 
large CBIs $(p=0.006)$. In small CBIs, vertebral artery dominance was identified in $15 / 31$ $(48 \%)$ of the patients, and significantly more often ipsilesional $(10 / 15,67 \%)$ than in large CBIs $(8 / 29,28 \%)(p=0.01)$.

Cardioembolism was the presumed aetiology in $37 \%$ of the patients, but there was no significant lobar predilection in those with small CBI. Six cases were cryptogenic. There was no significant difference either in the frequency of moderate-to-severe WML between those with small CBI $(17 / 32,53 \%)$ and those with large $(27 / 59,46 \%)$ CBI or in the severity of supratentorial WML with presenting clinical symptoms or signs. Overall, functional independence (mRS score $0-2$ ) at 3 months occurred in 27/33 (82\%) of the patients with small CBI and $42 / 73$ (58\%) of the patients with large CBI. A good outcome was more common among those with small CBI than among those with large CBI, after adjustment for age (OR 3.97, 95\% CI 1.41-11.15; $p=0.01$ ). There was no significant difference in 3-month outcomes between CBI involving the PICA and CBI involving the SCA. However, patients with CBI involving two or more cerebellar vascular territories showed a trend towards greater functional dependence at 3 months $(p=0.056)$.

Mortality at 3 months was 3/33 (9\%) for small CBI and 15/73 (21\%) for large CBI ( $p=$ 0.15). There was no significant difference in functional outcome between small CBI of the anterior and posterior lobes. The presence of moderate-to-severe WMLs on MRI correlated with functional dependence at 3 months, after adjustment for age (OR 3.47, 95\% CI 1.12-10.7; $p=0.03)$. This finding was significant for the patients with large CBI $(p=0.01)$ but not for those with small CBI $(p=0.7)$.

\section{Discussion}

In our large series of acute stroke due to CBI, small CBI accounted for approximately onethird of the cases and was most common with additional infarction in other brain regions, which is consistent with the literature $[13,14]$. Patients with small CBI were less symptomatic, and the infarcts were particularly located in the posterior cerebellar lobe. Small CBI was most often due to cardioembolism and significant VBD, with the latter more likely to cause large CBI. Although there was no association of supratentorial WML with presenting neurological symptoms and signs or the size of CBI, the extent of this disease predicted functional dependence at 3 months in those with large CBI.

Infarcts in the PICA territory generally correspond to the posterior lobe, and those in the SCA territory to the anterior lobe [15]. We found that small CBI was most frequently located in the posterior lobe, which is possibly related to the larger size and vascular supply [15]. The correlation of dizziness and limb ataxia with CBI in the anterior lobe is consistent with the sensorimotor function of this lobe $[5,16,17]$. Clinicoradiological studies of patients with focal cerebellar pathology including CBI found that cerebellar motor impairment typically correlates with lesions in the anterior lobe [17]. The topographical organisation may explain why small CBI, as in our series, often presents with non-specific symptoms and signs, or an asymptomatic MRI abnormality [18].

There was no difference in vascular risk factors and presumed cardioembolic aetiology between small and large CBI in our study, which is also consistent with the literature [3], and we were unable to confirm an association of cerebral small vessel disease with small CBI [5, 9]. Other studies have found cardioembolism and VBD to be the main causes of small CBI [3, 4]. In our study, a cardioembolic aetiology was present in $37 \%$ of the patients with small CBI, and this was seen with infarctions located in the anterior and posterior lobes. These findings indicate that patients with acute small CBI require investigation for a cardiac source of embolism. 
Our study identified a diversity of angiographic abnormalities in small CBI, including 2 cases of vertebral artery dissection. Large CBI was more commonly contralateral to a dominant vertebral artery, supporting the concept that posterior circulation thromboembolism is likely to have a greater impact on blood flow on the side of a non-dominant vertebral artery [19]. Atheromatous VBD was more frequent in large CBI, which is consistent with a propensity for occlusion of a proximal artery to compromise a larger territory of the cerebellum. Patients with small CBI may have underlying atheromatous $\operatorname{VBD}[9,20]$ that warrants vascular risk factor assessment and management.

It is intuitive that small CBI should have a better prognosis than large CBI, as is evident in our study. However, our finding of the severity of supratentorial WML being related to functional outcome at 3 months in large but not small CBI is consistent with another study showing associations between isolated CBI, age-related WML, and function 14 days after stroke onset [10]. These data suggest that the integrity of the supratentorial networks influences functional recovery, particularly with large CBI [10].

A limitation of our study is the small number of patients with isolated small CBI, although the size of the study is comparable to other studies $[4,9,10,16,20]$. Other issues are that we used a simple visual rating rather than a quantitative measure of WML, and an assessment of clinical outcome using the mRS rather than a cerebellum-specific functional measure [16].

In summary, small CBI, most often involving the posterior lobe, accounts for one-third of CBIs, and causes fewer symptoms and signs and has a better prognosis than large CBI. A low supratentorial WML burden is associated with better prognosis for patients with large CBI. Patients with small CBI require assessment of vascular risk factors and investigation for a cardioembolic source.

\section{Acknowledgements}

The authors wish to acknowledge the support of Assoc. Prof. Roy Beran, John Worthington, and Suzanne Hodgkinson, and Drs. Ho Choong, Neil Griffith, Ibrahim Hanna, Alan McDougall, and Daniel Wardman of the Neurology Department at Liverpool Hospital.

\section{Disclosure Statement}

The authors have no conflicts of interest to disclose.

\section{Funding Sources}

Z.C. undertook this work with funding from the UNSW (Australian Postgraduate Award) and Ingham Institute for Applied Medical Research.

\section{References}

1 Calic Z, Cappelen-Smith C, Anderson CS, Xuan W, Cordato DJ: Cerebellar infarction and factors associated with delayed presentation and misdiagnosis. Cerebrovasc Dis 2016;42:476-484.

2 Kase CS, Norrving B, Levine SR, Babikian VL, Chodosh EH, Wolf PA, et al: Cerebellar infarction. Clinical and anatomic observations in 66 cases. Stroke 1993;24:76-83.

3 Amarenco P, Levy C, Cohen A, Touboul PJ, Roullet E, Bousser MG: Causes and mechanisms of territorial and nonterritorial cerebellar infarcts in 115 consecutive patients. Stroke 1994;25:105-112. 
4 Amarenco P, Kase CS, Rosengart A, Pessin MS, Bousser MG, Caplan LR: Very small (border zone) cerebellar infarcts. Distribution, causes, mechanisms and clinical features. Brain 1993;116(pt 1):161-186.

5 De Cocker LJ, van Veluw SJ, Fowkes M, Luijten PR, Mali WP, Hendrikse J: Very small cerebellar infarcts: integration of recent insights into a functional topographic classification. Cerebrovasc Dis 2013;36:81-87.

6 Stoodley CJ, Schmahmann JD: Evidence for topographic organization in the cerebellum of motor control versus cognitive and affective processing. Cortex 2010;46:831-844.

7 Debette S, Markus HS: The clinical importance of white matter hyperintensities on brain magnetic resonance imaging: systematic review and meta-analysis. BMJ 2010;341:c3666.

$\checkmark 8$ Liou LM, Chen CF, Guo YC, Cheng HL, Lee HL, Hsu JS, et al: Cerebral white matter hyperintensities predict functional stroke outcome. Cerebrovasc Dis 2010;29:22-27.

-9 Min WK, Kim YS, Kim JY, Park SP, Suh CK: Atherothrombotic cerebellar infarction: vascular lesion-MRI correlation of 31 cases. Stroke 1999;30:2376-2381.

10 Grips E, Sedlaczek 0, Bäzner H, Fritzinger M, Daffertshofer M, Hennerici M: Supratentorial age-related white matter changes predict outcome in cerebellar stroke. Stroke 2005;36:1988-1993.

11 Schmahmann JD, Doyon J, McDonald D, Holmes C, Lavoie K, Hurwitz AS, et al: Three-dimensional MRI atlas of the human cerebellum in proportional stereotaxic space. Neuroimage 1999;10:233-260.

12 Fazekas F, Chawluk JB, Alavi A, Hurtig HI, Zimmerman RA: MR signal abnormalities at 1.5 T in Alzheimer's dementia and normal aging. AJR Am J Roentgenol 1987;149:351-356.

13 Canaple S, Bogousslavsky J: Multiple large and small cerebellar infarcts. J Neurol Neurosurg Psychiatry 1999; 66:739-745.

14 Terao S, Miura N, Osano Y, Takatsu S, Adachi K, Noda A, et al: Multiple cerebellar infarcts: clinical and pathophysiologic features. J Stroke Cerebrovasc Dis 2005;14:193-198.

15 De Cocker LJ, Geerlings MI, Hartkamp NS, Grool AM, Mali WP, van der Graaf Y, et al: Cerebellar infarct patterns: the SMART-Medea study. Neuroimage Clin 2015;8:314-321.

16 Schmahmann JD, Macmore J, Vangel M: Cerebellar stroke without motor deficit: clinical evidence for motor and non-motor domains within the human cerebellum. Neuroscience 2009;162:852-861.

17 Schoch B, Dimitrova A, Gizewski ER, Timmann D: Functional localization in the human cerebellum based on voxelwise statistical analysis: a study of 90 patients. Neuroimage 2006;30:36-51.

-18 De Cocker LJ, Kloppenborg RP, van der Graaf Y, Luijten PR, Hendrikse J, Geerlings MI, et al: Cerebellar cortical infarct cavities: correlation with risk factors and MRI markers of cerebrovascular disease. Stroke 2015;46: 3154-3160.

19 Hong JM, Chung CS, Bang OY, Yong SW, Joo IS, Huh K: Vertebral artery dominance contributes to basilar artery curvature and peri-vertebrobasilar junctional infarcts. J Neurol Neurosurg Psychiatry 2009;80:1087-1092.

-20 De Cocker LJ, Compter A, Kappelle LJ, Luijten PR, Hendrikse J, Van der Worp HB: Cerebellar cortical infarct cavities and vertebral artery disease. Neuroradiology 2016;58:853-857. 